\title{
Experimental study of a high intensity radio-frequency cooler
}

\author{
Ramzi Boussaid, G. Ban, and J. F. Cam \\ LPC-IN2P3, ENSICAEN, 6 Boul. Maréchal Juin, 14050 Caen, France
}

(Received 20 December 2014; published 9 July 2015)

\begin{abstract}
Within the framework of the DESIR/SPIRAL-2 project, a radio-frequency quadrupole cooler named SHIRaC has been studied. SHIRaC is a key device of SPIRAL-2, designed to enhance the beam quality required by DESIR. The preliminary study and development of this device has been carried out at Laboratoire de Physique Corpusculaire de CAEN (LPC Caen), France. The goal of this paper is to present the experimental studies conducted on a SHIRaC prototype. The main peculiarity of this cooler is its efficient handling and cooling of ion beams with currents going up as high as $1 \mu \mathrm{A}$ which has never before been achieved in any of the previous coolers. Much effort has been made lately into these studies for development of appropriate optics, vacuum and rf systems which allow cooling of beams of large emittance ( $~ 80 \pi \mathrm{mm} \mathrm{mrad}$ ) and high current. The dependencies of SHIRaC's transmission and the cooled beam parameters in terms of geometrical transverse emittance and the longitudinal energy spread have also been discussed. Investigation of beam purity at optimum cooling condition has also been done. Results from the experiments indicate that an emittance reduction of less than $2.5 \pi \mathrm{mm} \mathrm{mrad}$ and a longitudinal energy spread reduction of less than $4 \mathrm{eV}$ are obtained with more than $70 \%$ of ion transmission. The emittance is at expected values whereas the energy spread is not.
\end{abstract}

DOI: 10.1103/PhysRevSTAB.18.072802

PACS numbers: 29.27.Fh, 29.27.Eg, 07.30.-t, 07.50.-e

\section{INTRODUCTION}

In the context of generation of newly developed nuclear facilities based on the ISOL method [1] for the production of rare and exotic ion beams with intensities up to $1 \mu \mathrm{A}$ and emittances up to $80 \pi \mathrm{mm}$ mrad [2], the SPIRAL-2 project is being installed at GANIL laboratory in France [3-5]. Ion beams produced by SPIRAL-2 will subsequently suffer from isobaric contaminations [6].

The Désintégration, Excitation et Stockage d'Ions Radioactifs (DESIR) facility is a low energy beam experiment meant to receive beams from SPIRAL-2 $[7,8]$. The high degree of purity which is required to have the highest limits of stability will be achieved by the implementation of a high resolution separator (HRS) $[9,10]$ in the SPIRAL-2 production building.

In order to use the produced beams in the best possible way, the HRS must receive beams of low transverse emittance $(\leq 3 \pi \mathrm{mm} \mathrm{mrad})$ and low energy spread $(\sim 1 \mathrm{eV})$. Currently, there are several instruments which are operating at the world's radioactive beam installations and are capable of providing low beam emittances with good efficiency. For this purpose, a radio-frequency quadrupole cooler (RFQC) should be installed in front of the HRS. The RFQCs are based on a combination of a

\footnotetext{
*boussaidramzii@gmail.com

Published by the American Physical Society under the terms of the Creative Commons Attribution 3.0 License. Further distribution of this work must maintain attribution to the author(s) and the published article's title, journal citation, and DOI.
}

radio-frequency quadrupole (RFQ) filled with a buffer gas, and an axial field to guide the beam. The ions are first electrostatically retarded to a kinetic energy of a few $\mathrm{eV}$ before entering the RFQ structure. Once inside, the rf fields provide radial confinement while an additional dc guiding electric field pulls the ions slowly through the buffer gas. Interactions with the buffer gas atoms reduce the kinetic energy of the ions and thereafter enhance the beam quality. Beam quality is here used in the broad sense of transverse emittance and longitudinal energy spread.

The RFQCs are already used in several projects, such as LEBIT at NSCL [11], ISCool at ISOLDE [12,13], and TITAN at TRIUMF [14]. Unlike previous RFQCs, which can cool and handle only beams of low intensities $(\sim 100 \mathrm{nA})$ and small emittances $(\sim 10 \pi \mathrm{mm} \mathrm{mrad})$, the RFQC required for SPIRAL-2 must be able to capture higher intensities and larger emittance of the incoming beam. In order to fully exploit these increases in intensity, a new RFQC prototype named Spiral-2 High Intensity Radiofrequency Cooler (SHIRaC) was developed and tested at the LPC-Caen laboratory at France. This prototype is designed to serve dual functions, providing desirable beam quality for the HRS and transmitting more than $60 \%$ of the incoming ions toward the HRS.

SHIRaC needs to get over several challenges in order to handle SPIRAL-2 beams, owing to the following three systems: an appropriate optics system for capturing large emittances, an rf system which provides highest rf voltage amplitude to overcome the space charge effects resulting due to the high beam currents [15] and a specific vacuum system to control the buffer gas pressure. 
Numerical simulations enabling to optimize SHIRaC's design and to determine the needed cooling parameters in terms of buffer-gas pressure, rf parameters, electrode voltages, etc., had been published already [16].

The development and tests conducted on SHIRaC's systems are presented in this paper. The results of the experimental investigations of the $\mathrm{Cs}$ ion beam cooling with beam currents up to $1 \mu \mathrm{A}$ are also reported. The dependencies of the ion transmission and the cooled beam properties on space charge effects, buffer gas pressure and rf voltage amplitude are outlined. Beam purity at the cooling optimum conditions will also be investigated.

\section{EXPERIMENTAL SETUP}

RFQCs currently play an important role in purifying and preparing radioactive ion beam in today's installations. Designing RFQC's is a complex process including several aspects of physics as well as technical aspects. The research reported here should help determine the needed HRS specifications, as previously mentioned. In the SPIRAL2 context, SHIRaC consists of three systems: optics system, vacuum system and electronic system.

\section{A. SHIRaC optics}

Since the main purpose of the SHIRaC project is improving the SPIRAL-2 beam quality, therefore special attention needs to be taken in the design of the optics system.

Similar to many coolers currently used worldwide, such as those presented in Refs. [11,17-19], SHIRaC's optical structure can be divided into three parts as shown in Fig. 1: injection and deceleration, RFQ, and extraction and reacceleration. The optical design of all three parts was optimized using SIMION-3D v8.0 software [20].

The injection part contains electrodes for decelerating the ions down to input energy into RFQ. This energy should be within the expected range (100-200 eV) [17]; in the present case, it is approximately $140 \mathrm{eV}$. The beam is injected into the RFQ chamber through the injection plate. A lens of three electrodes is mounted to avoid any ion losses during the deceleration process and to improve their injection.
The RFQ part is the main RFQC chamber. It encloses the buffer gas and the RFQ. This part is devoted to confine the injected beam efficiently and then to cool it progressively. In order to adapt to SPIRAL2/DESIR authentic beams, the internal radius value is set to $r_{0}=5 \mathrm{~mm}$. The RFQ's electrodes are segmented into 18 equidistant segments of $40 \mathrm{~mm}$ length each. This segmentation enables creating a longitudinal electric field to guide the cooled ions which will be then released from the RFQ limit through extraction plate.

The extraction and reacceleration part contains the electrodes that make the ion beam extract and then accelerate the cooled ions up to their initial energy.

Details of design and electrode dimensions are described in Refs. [16,21].

\section{B. Vacuum system}

To run an RFQC, we must introduce a buffer gas inside an RFQ chamber that helps cool the ion beam. The cooling is provided subsequent to successive collisions between trapped ions and buffer gas atoms inside the RFQ chamber. For optimum cooling, buffer gas pressures of a few $\mathrm{Pa}$ are required [22].

The vacuum system of an RFQC in general, and of SHIRaC in particular, faces the main challenge of keeping high vacuum at the injection and extraction parts whilst adding a high gas load into the RFQ chamber. It consists of separate sections connected by exchangeable apertures where various pressures distribution are obtained by differential pumping $[13,21]$. The differential pumping system is very crucial for achieving optimal cooling and transmission efficiency but cannot avoid the gas leakage through entrance and exit apertures. The leakage of gas causes the degradation of the beam quality and loss of ions that happen due to the scattering with the residual gas. These harmful effects can be reduced by careful design of the differential pumping system and by the optimization of the dimension of the injection and extraction plates.

The main goal is to reach pressures lower than $10^{-2} \mathrm{~Pa}$ inside the injection and extraction parts, and lower than $10^{-3} \mathrm{~Pa}$ in the rest of the beam line. For this purpose,

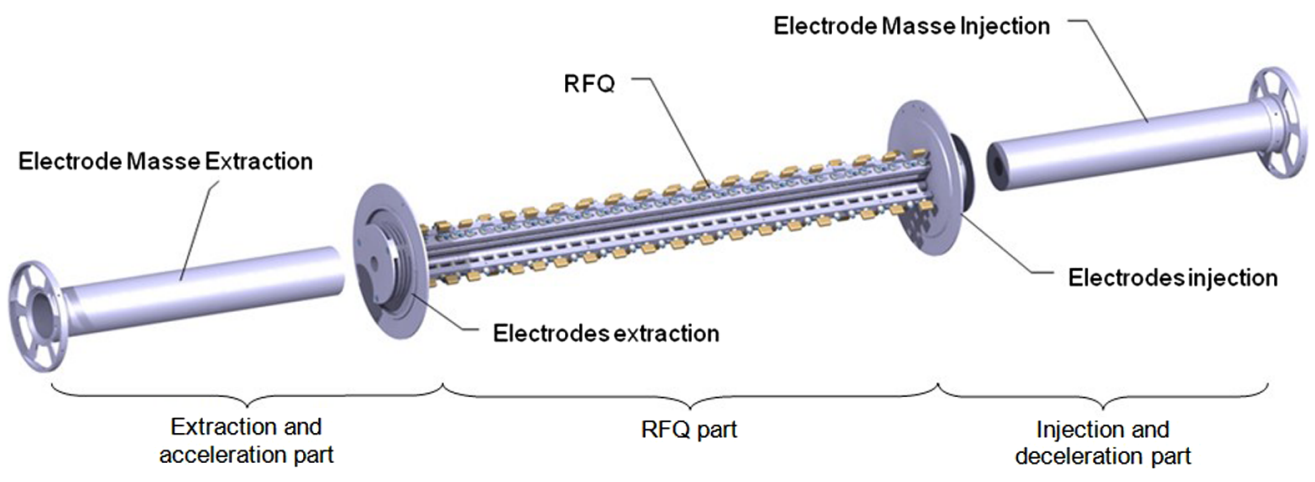

FIG. 1. Schematic of the SHIRaC optics system: Design of the three parts of SHIRaC. 


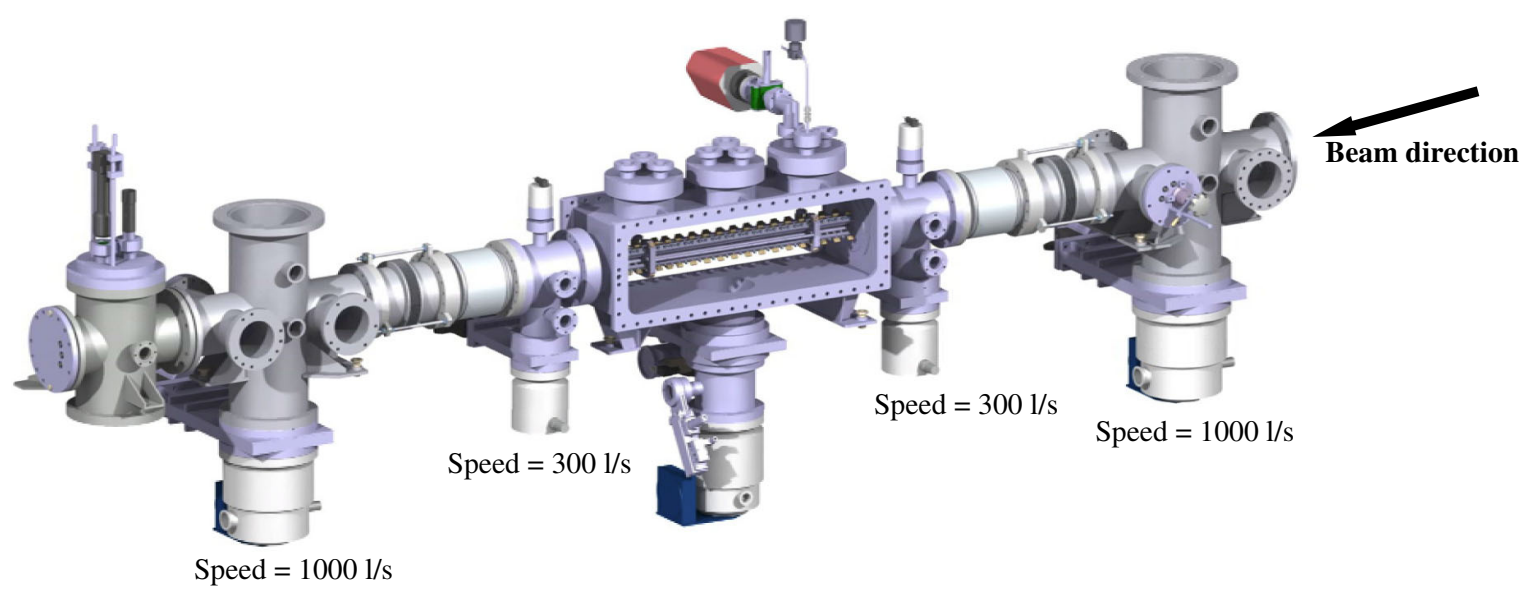

FIG. 2. 3D layout of the differential pumping vacuum system of SHIRaC.

injection and extraction plates have holes of dimensions 6 and $4 \mathrm{~mm}$, respectively, and $2 \mathrm{~mm}$ size. Five turbomolecular pumps with different pumping speeds are also installed (Fig. 2).

Table I shows pressure measurements inside the different parts of SHIRaC beam line with various RFQ pressures (pressure into the RFQ chamber). We note, nonetheless, the needed environment pressure is provided by this system.

\section{Electronic system}

For achieving the rf quadrupole field into the RFQ, it was necessary to feed in two opposite phase sinusoidal rf voltages to the segment pairs. This resulted in all the segments running together as four continuous quadrupole rods. The RFQC requires applying an rf voltage, of a few $\mathrm{kV}$ with a few $\mathrm{MHz}[15,16,23]$, to the electrodes. To evaluate the sustainability of necessary rf voltage in the operational buffer gas pressure range, we developed a new rf system (Fig. 3). For the present work, a relatively strong rf confinement voltages are needed to overcome the spacecharge effects $[22,24,25]$. Therefore, in order to achieve high rf potentials across the trap electrodes at reasonable power levels, some form of resonating circuit must be provided. For the frequency range required by SHIRaC and the load levels presented, the only feasible resonant circuit is a lumped LC split series based on an Inductor-Capacitor resonating circuit with a transformer [26]. With such circuits, coupling between the function generator and LC circuit is done using a ferrite core transformer. Due to this material saturation $[13,26]$ the maximum allowable rf parameters are at about $1 \mathrm{kV}$ and $1 \mathrm{MHz}$ [23]. For the high-voltage operation required in the present work, and in order to prevent failures caused by the saturation of the transformer core, it is prudent to use a resonant LC circuit with air-inductive coupling (Fig. 3) where the coupling is done by a single turn injected into the hollow coils of the LC circuit. The LC circuits are composed of a parallel air induction system single coil and a resonant LC circuit: two hollow coils (L1 and L2) and one variable capacitor (CG $35-1000$ pF), as shown in Fig. 3.

The rf system is a critical instrument of RFQC because the electric breakdown [22] and the burning ceramic insulator can occur into the RFQ chamber for a few $\mathrm{kV}$ of $\mathrm{rf}$ amplitudes. To avoid the burning, the whole internal structure is done using polyether ether ketone (PEEK), as shown in Fig. 4. As we have seen previously, for allowing the ions to pass through the RFQ, it was necessary, on top of the rf voltage, to couple a dc bias onto each segment pair, the bias level being determined by the segment pair's location along the axis.

Results of rf voltages on the quadrupole's electrodes when plotted as a function of the generator's input voltage and with a LC resonant circuit of eight turns per hollow coils, are shown in Fig. 5. Up to maximum allowed $500 \mathrm{mV}$ input generator voltage, it is observed for sure that the $\mathrm{rf}$ circuit does not show any signs of being saturated and output voltage ( $\mathrm{rf}$ voltage) can go even higher if any increase of the input voltage occurs. This behavior shows

TABLE I. Pressure measurements inside SHIRaC beam line sections: the source section, the injection section, the RFQ chamber, the extraction section and the target region.

\begin{tabular}{lccccc}
\hline \hline $\begin{array}{l}\text { Gas rate } \\
(\mathrm{ml} / \mathrm{mn})\end{array}$ & $\begin{array}{c}\text { Pressure in target } \\
\text { region }(\mathrm{Pa})\end{array}$ & $\begin{array}{c}\text { Pressure in extraction } \\
\text { section }(\mathrm{Pa})\end{array}$ & $\begin{array}{c}\text { Pressure in RFQ } \\
\text { section }(\mathrm{Pa})\end{array}$ & $\begin{array}{c}\text { Pressure in injection } \\
\text { section }(\mathrm{Pa})\end{array}$ & $\begin{array}{c}\text { Pressure in source } \\
\text { section }(\mathrm{Pa})\end{array}$ \\
\hline 30 & $2.0 \times 10^{-3}$ & $9.1 \times 10^{-3}$ & 3.1 & $2.1 \times 10^{-2}$ & $3.5 \times 10^{-3}$ \\
25 & $1.5 \times 10^{-3}$ & $6.6 \times 10^{-3}$ & 2.5 & $1.6 \times 10^{-2}$ & $2.8 \times 10^{-3}$ \\
20 & $1.3 \times 10^{-3}$ & $5.4 \times 10^{-3}$ & 2.1 & $1.2 \times 10^{-2}$ & $2.3 \times 10^{-3}$ \\
\hline \hline
\end{tabular}



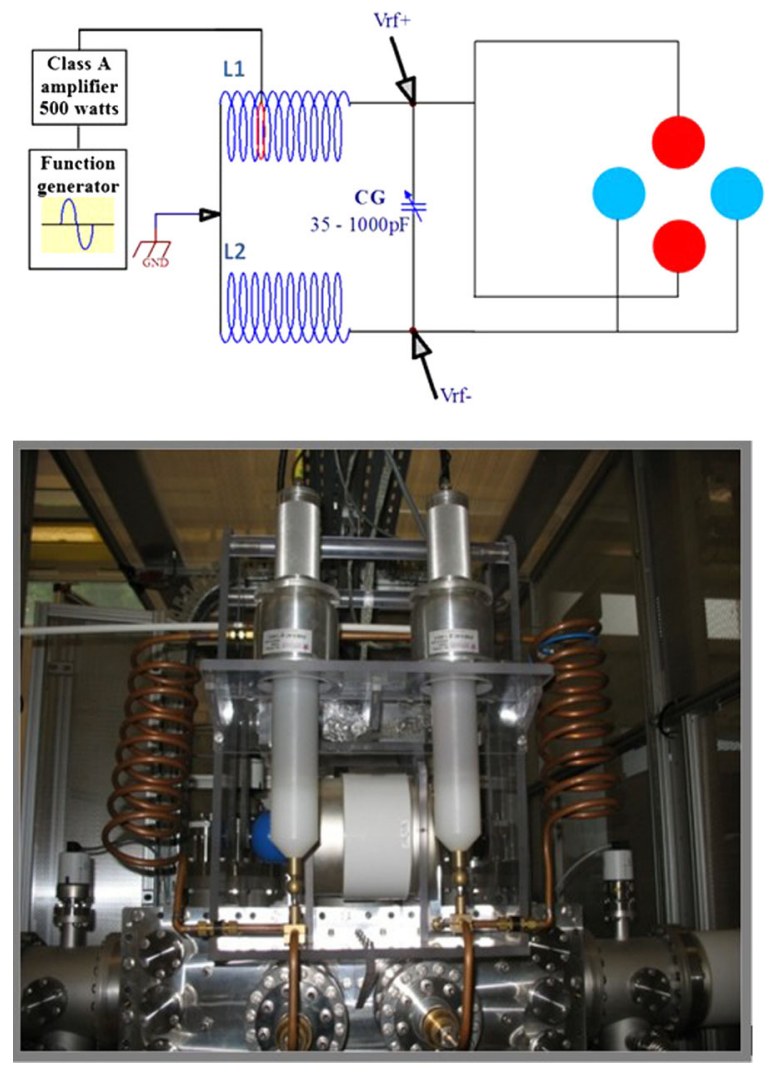

FIG. 3. Design of the rf electronic system (top) and the real $\mathrm{rf}$ system of SHIRaC (bottom).

the efficiency of such air-inductor coupling to produce higher rf voltage in comparison with air-cores coupling. Thanks to the air-inductor coupling, such a circuit can operate quite comfortably even while delivering rf voltages up to $8 \mathrm{kV}$ and $5 \mathrm{MHz}$ on either side of the hollow coil. Furthermore, introducing helium into the RFQ chamber did not cause any noticeable problems until pressures approaching $5 \mathrm{~Pa}$.

The reduction of the cooling process degradation, caused by the rf heating [22,27], requires highest resonating frequencies $f_{0}$ [16]. For the above stated reason, inductance of hollow coils had to be reduced. This was accomplished

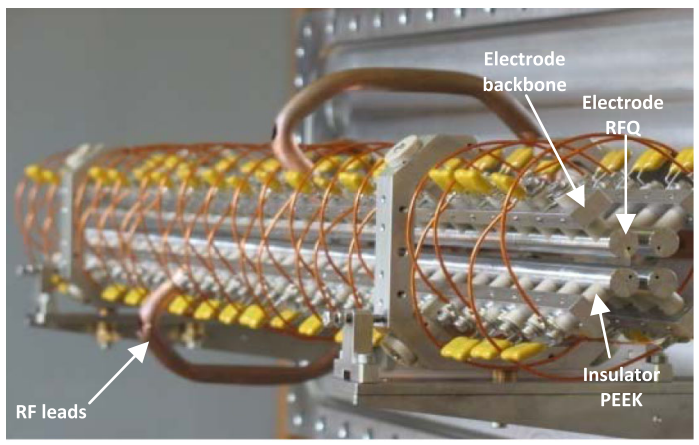

FIG. 4. The voltages delivery scheme to the quadrupole electrodes of the rf supply and the power supplies for the dc axial guiding voltage.

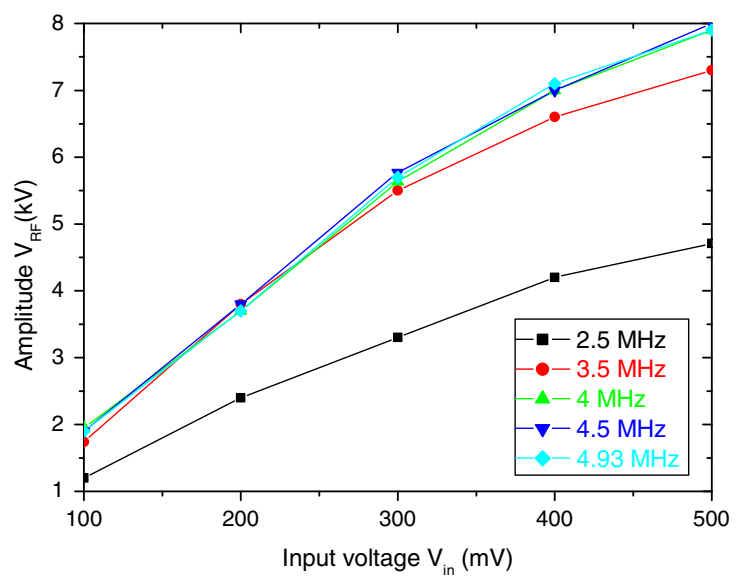

FIG. 5. Plot of the rf voltage amplitudes as a function of the input voltages for various frequencies set in the function generator and for a configuration of eight turns per hollow coil.

by testing the influence of the number of turns per coil on the resonating frequency (Table II). The $\mathrm{rf}$ performance improvements are perceptible, as illustrated in the table. The resonating frequencies get enhanced and reach about $9 \mathrm{MHz}$.

\section{EXPERIMENTAL RESULTS}

In order to confirm the performance of SHIRaC as predicted by the simulations works [16] and to enhance the cooled beam quality, we have completed several experimental studies based on the specifications of this project. For that purpose, we have used an IGS-4 ionization surface source which can provide ${ }^{133} \mathrm{Cs}^{+}$ion beams with intensities of up to $1 \mu \mathrm{A}$ and energy of up to $5 \mathrm{keV}$. Such beams have emittances of a few tens $\pi \mathrm{mm}$ mrad and energy spread of a few tens of $\mathrm{eV}$. We will be focusing our attention on the effects of the space charge, the rf voltage and the buffer gas pressure on the ion transmission and beam quality.

Tests of SHIRaC have been performed at LPC-Caen (France). A sketch of the experimental line is shown in Fig. 6; the RFQ chamber is preceded with the injection part connecting it to an ionization surface source. The chamber is followed by an extraction beam part equipped with beams diagnostics for current and beam measurements. The rf electronic system is mounted behind this chamber.

The experimental test has been performed with an $\mathrm{rf}$ frequency $f_{0}$ of $4.5 \mathrm{MHz}$. Given that the rf voltage amplitude $V_{\mathrm{rf}}$ and the Mathieu parameter $\mathrm{q}$ are proportional [28,29]:

TABLE II. Resonating frequencies of rf voltages with various numbers of turns per coils.

\begin{tabular}{lc}
\hline \hline Number of turns per coils & $\boldsymbol{f}_{0}(\mathrm{MHz})$ \\
\hline 2 & 9 \\
5 & 6,5 \\
8 & 5 \\
\hline \hline
\end{tabular}




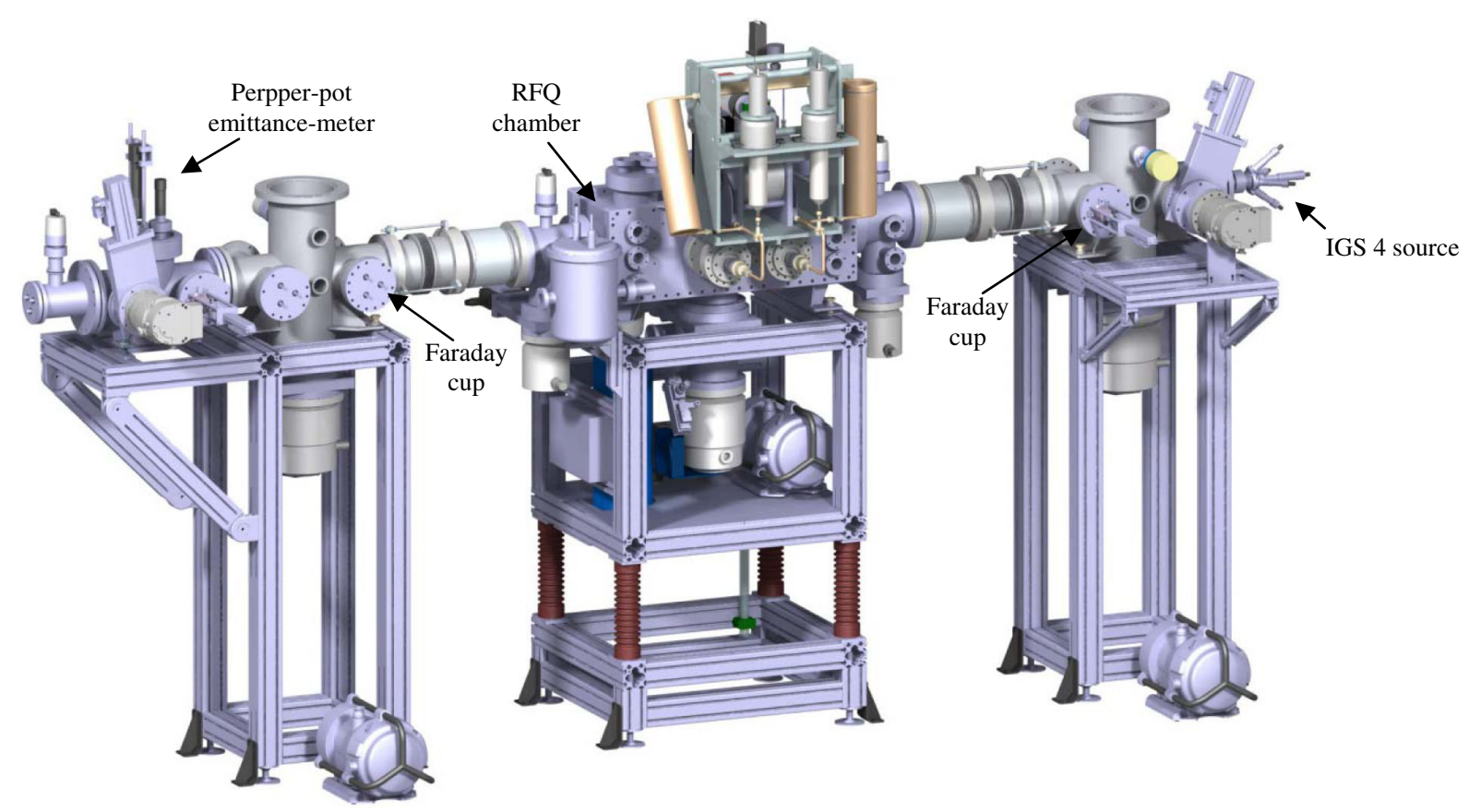

FIG. 6. 3D schematic of the planned test setup for the SHIRaC prototype.

$$
V_{\mathrm{rf}}=\frac{m r_{0}^{2} \pi^{2} f_{0}^{2}}{\mathrm{e}} q
$$

where e, $m$ and $r_{0}$ are, respectively, the elementary charge, the cesium ion mass and the quadrupole inner radius. It will subsequently be relevant to study the measurement dependencies on the parameter q rather than the rf amplitude. Under the same conditions stated above, this proportionality is of 6.9 for the cesium case.

\section{A. Transmission efficiency}

In order to characterize SHIRaC, our first concern is to find the operating parameters via the transmission efficiency. The operating parameters point to an appropriate input energy (the energy the ions have when they enter the RFQ), a buffer gas pressure and a guiding dc voltage. All of them enable to provide an optimum cooling, corresponding to the maximum ion transmission. The transmission is the ratio of the beam current after the extraction section by the beam current at the injection section, measured by a cup Faraday.

Tests were performed and showed that the operating parameters are $140 \mathrm{eV}$ input energy, $2.5 \mathrm{~Pa}$ buffer gas pressure and $16 \mathrm{~V} / \mathrm{m}$ guiding dc electric field [21]. In Sec. III B, we show that under such conditions, the cooled beam possesses a high purification level.

Simulation studies led us to conclude that the influences of the space charge and rf voltage amplitude on the ion transmission are of major importance [16]. This induces us to study the dependencies of SHIRaC transmission on the beam currents and rf voltage amplitudes. The transmission behavior in dependence upon the beam current was investigated, as shown in Fig. 7. It gradually decreases with beam currents and remains above $70 \%$ for currents going up to $1 \mu \mathrm{A}$. This low loss of ions shows the ability of the optics system to capture more than $93 \%$ of incoming ions and explains the ability of high rf voltage amplitude to overcome the space charge effect and to confine such ions along the RFQ.

The transmission dependence on the rf voltage (i.e., the Mathieu parameter $q$ ) is communally called ion stability diagram. Figure 8 reveals this stability while the ions can reach the RFQ exit for q between 0 and 0.9 [30]. As is clear from the figure, transmission increases with an increase in $\mathrm{q}$

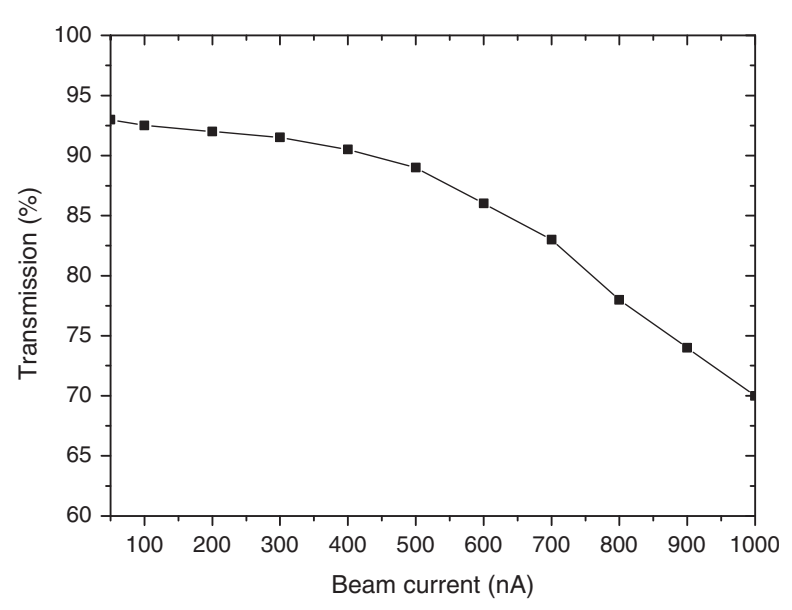

FIG. 7. Space charge effect on the ion transmission: variation of the transmission versus beam current for $2.5 \mathrm{~Pa}$ buffer gas pressure. 


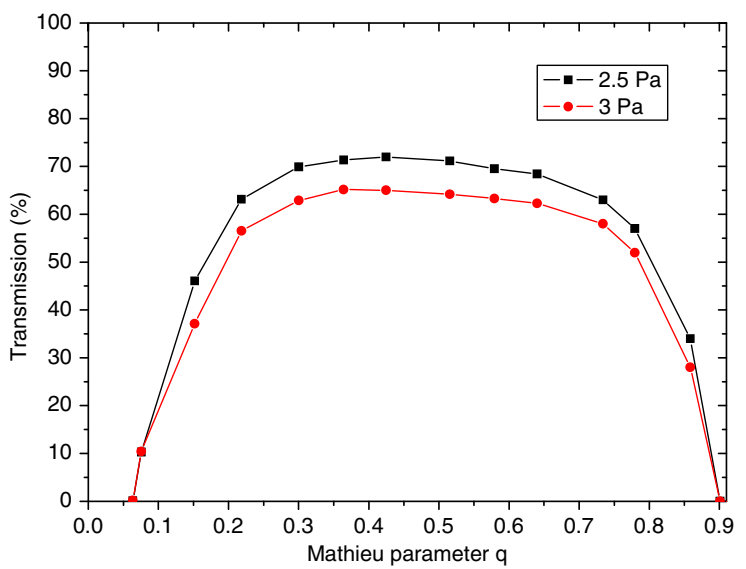

FIG. 8. Stability diagram at $1 \mu \mathrm{A}$ beam current: variation of the transmission as a function of the Mathieu parameter $\mathrm{q}$ for various buffer gas pressure.

and a maximum is reached at $\mathrm{q}$ between 0.3 and 0.5 ; thereafter a decrease is observed. This transmission behavior is explained by the existence of a threshold of potential, corresponding to the maximum transmission, at which the confining force is sufficient to overcome the defocusing effects such as the gas diffusion and space charge. The relative difference between the red curve and the black curve once again highlights that the optimum cooling is obtained with an RFQ pressure of $2.5 \mathrm{~Pa}$.

According to Ref. [31], a widening phenomenon of the stability diagram can occur for high beam currents. For the present study, this phenomenon is not present for beam current going up to $1 \mu \mathrm{A}$.

\section{B. Cooled beam purity}

To ascertain any possible exchange of ionization between cesium ions and buffer gas or with possible impurities [32], cooled ion beam purity was determined. For that, we have mounted a voltage switcher on the first electrode of the extraction lens, allowing to extract cooled beam in bunch mode, and a MCP (Micro Channel Plate) detector was placed after the extraction section. The detector consists of an Einzel lens, an attenuator and a MCP screen [Fig. 9 (top)]. As each ion reaches the MCP detector plane, its arrival time is binned appropriately, creating a time of flight histogram for each extracted ensemble that simulates the experimental ion signal recorded by the MCP detector. These signals imitate the temporal distribution of extracted cooled beam. Since they are related to ion mass, therefore each peak of these signals will be associated to a set of ions of the same mass.

The measurement was assessed under optimum cooling conditions and the result is presented in Fig. 9 (bottom). Ions other than cesium were not detected and this confirms that there is no ionization exchange between cesium ions and buffer gas atoms or with the impurities of the buffer gas.
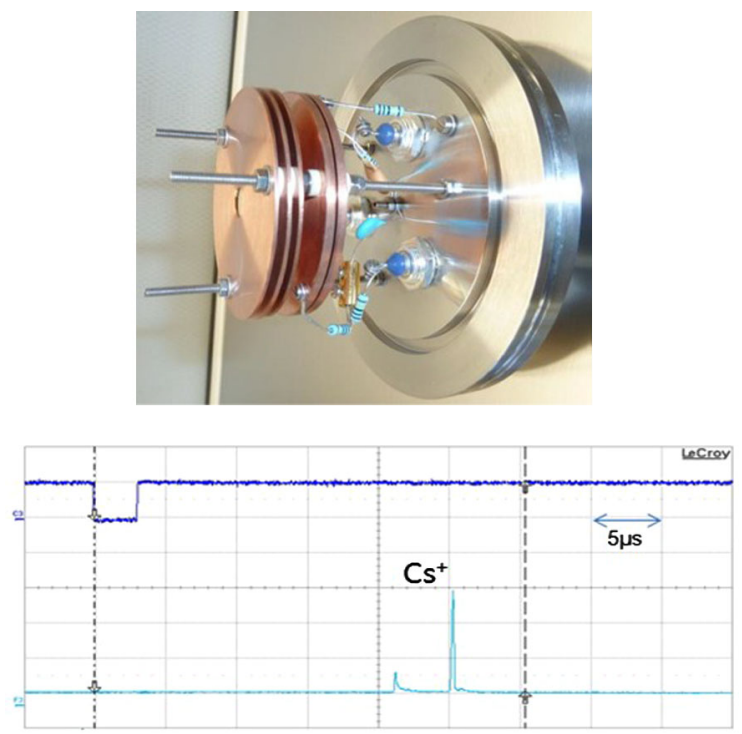

FIG. 9. An overview of the MCP detector (top) and cooled ions TOF spectrum for $1 \mu \mathrm{A}$ of beam current under the optimum cooling conditions (bottom).

\section{Geometric transverse emittance}

For determining the geometric transverse emittance, one kind of measurements has been carried out with a commercial pepper-pot Emittance meter [33] inserted perpendicularly to the beam direction and at the output of the beam line (Fig. 6).

The pepper-pot/multislit method is a common way to determine the geometric transverse emittance of ion beams. The pepper-pot mask consists of an even plate with a twodimensional array of holes in it. The part of the beam which passes the pepper-pot through its holes gets separated into several spots equidistantly, and thereafter hit a scintillating screen (MCP) located further downstream. The used pepper-pot has the dimensions shown in Table III.

The rms transverse emittance of a beam can be calculated from the position, size, and shape of these spots [34]. The geometric transverse emittance can then be deduced from the rms emittance where it is defined at $90 \%$ of the action volume [13].

To deduce equivalent emittance at $60 \mathrm{keV}$ of beam energy, we can use the following equation [13]:

$$
\varepsilon_{E_{2}}=\varepsilon_{E_{1}} \times \sqrt{\frac{E_{1}}{E_{2}}},
$$

TABLE III. Parameters and dimensions of the pepper-pot emittance meter.

\begin{tabular}{lc}
\hline \hline Hole-hole distance $(\mathrm{mm})$ & $1 \pm 0.01$ \\
Hole size $(\mu \mathrm{m})$ & $100 \pm 5$ \\
Mask-MCP distance $(\mathrm{mm})$ & $60 \pm 0.2$ \\
\hline \hline
\end{tabular}




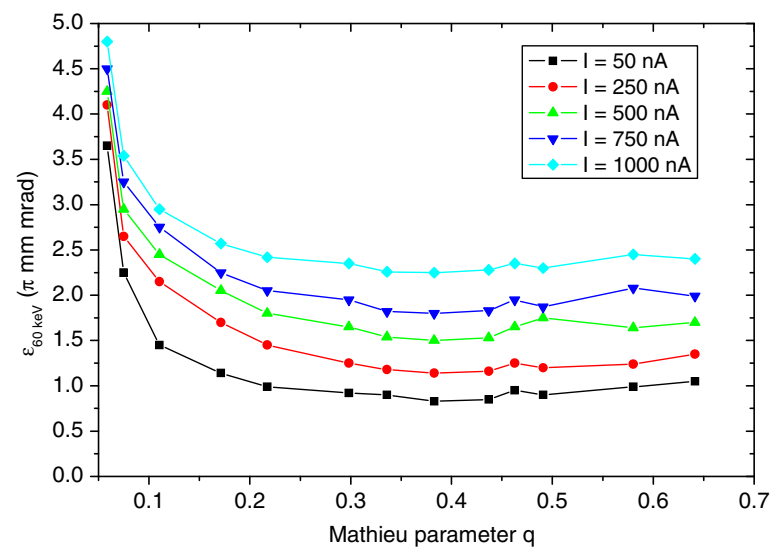

FIG. 10. Measurements of geometric transverse emittances versus Mathieu parameter $q$ (i.e., rf voltage) for various beam currents.

where $\varepsilon_{E_{1}}$ and $\varepsilon_{E_{2}}$ are the beam emittances at beam energies of $E 1$ and $E 2$, respectively.

With this emittance meter, it is possible to follow the transverse emittance evolution. Figure 10 shows the emittance behavior for different rf amplitudes (i.e., Mathieu parameter q) and for various beam currents. These measurements are clearly sensitive to both rf voltage and beam current simultaneously. It is observed that, for all beam currents, emittance first decreases to a minimum value as rf voltage increases, and then slightly increases as the rf voltage is further increased. The rapid decrease of the curves explains the effect of the rf voltage on confining the ions and subsequently on enhancing the beam quality. Afterwards, as rf voltage is increased, emittance starts to saturate for $q$ between $0.3-0.5$, which corresponds to maximum cooling power. For $\mathrm{q}$ greater than 0.5 , the emittance tends to increase slightly. This is because the rf heating effect arises for these values of $q[22,27]$.

The emittance is also seen to increase in proportion to the ion beam current, as expected from space charge considerations [16]. The optimum emittance values do not exceed $2.5 \pi \mathrm{mm} \mathrm{mrad}$ and are comparable to those for the previous coolers, notwithstanding the beam currents are often 10 times higher.

\section{Longitudinal energy spread}

Characterization of beam longitudinal energy spread in a space-charge dominated beam is very important in understanding the cooling process of intense beams. The measurements of the transmission variation as a function of the dc retarding voltage [Fig. 11 (top)], which is applied to the last segment of the RFQ, are used to determine the longitudinal energy spread $\Delta E$ using the width (FWHM) of its derivative [Fig. 11 (bottom)]. The energy spread reduction, showing the cooling effect, is illustrated in Fig. 11 (bottom) as regards its decrease with the increase of the PRFQ. Above $2.5 \mathrm{~Pa}$, an increase is seen and it is
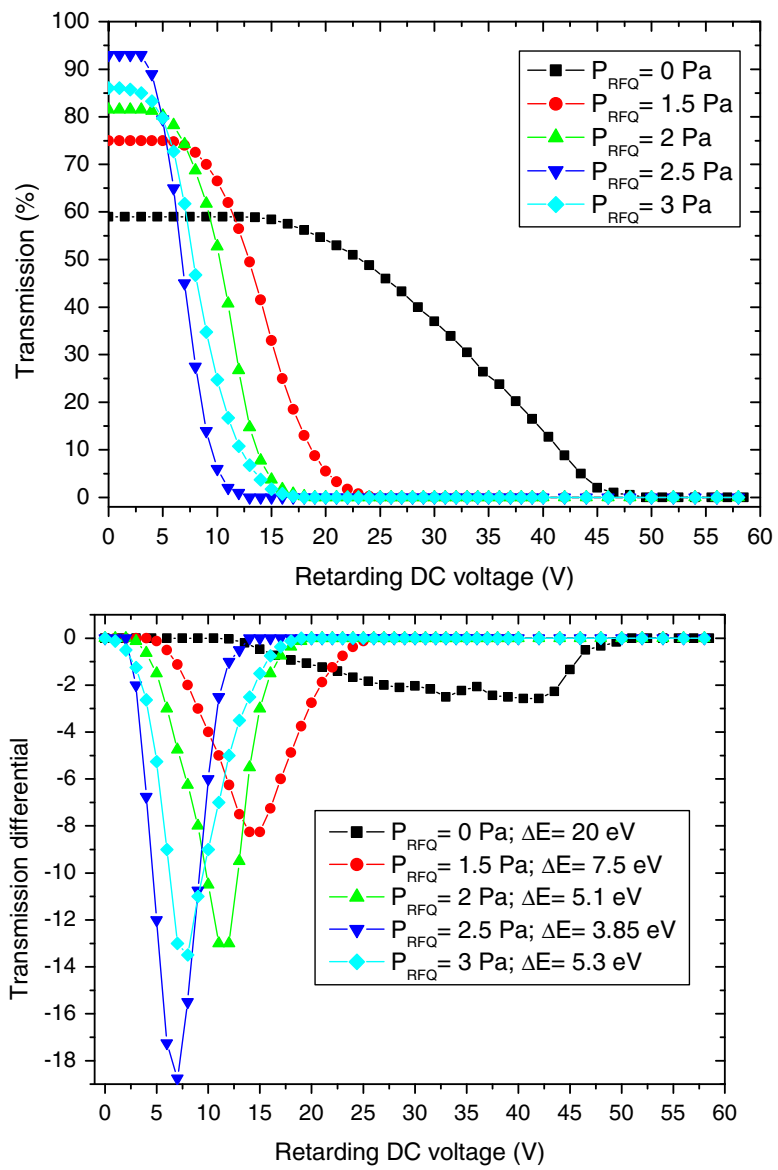

FIG. 11. Measurements of the longitudinal energy spread with various buffer gas pressure $P_{\mathrm{RFQ}}$ and for beam current of $50 \mathrm{nA}$ : variation of the transmission as a function of the retarding dc voltage (top) and variation of its differential versus the retarding dc voltage (bottom).

explained by the degradation of the cooled beam quality by the buffer gas diffusion at the RFQ exit. The lowest energy spread obtained with $2.5 \mathrm{~Pa}$ buffer gas pressure once again confirms the suitable choice of the optimum cooling conditions.

The simulation results presented in Ref. [16] have shown that space charge has an important degrading effect on the energy spread. Figure 12 reports quantitative results of this phenomenon regarding the growth of energy spread from 3.9 to about $6.5 \mathrm{eV}$ for beam currents going from $50 \mathrm{nA}$ up to $1 \mu \mathrm{A}$. These results are similar to those shown by the numerical simulations. This growth explains clearly the space charge effect. However the large energy spread arising at low beam current as well as at high beam current induces us to find the cause of this growth.

The main difference between previous RFQCs and SHIRaC lies in the rf voltage amplitude used, which is less than $1 \mathrm{kV}$ in the former compared to more than $2.5 \mathrm{kV}$ in the latter. This leads us to study the rf voltage effect on the energy spread. In Fig. 13, we present the variation of $\Delta E$ and its corresponding transmission as a function of the 


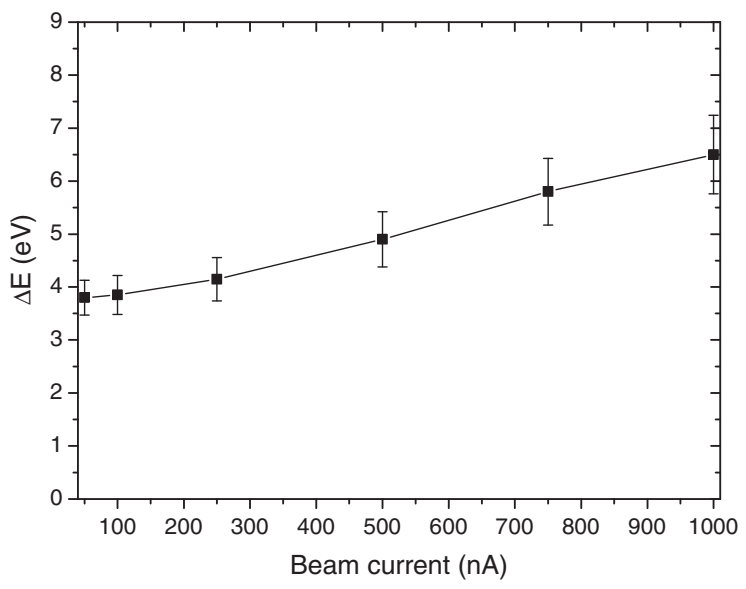

FIG. 12. Space charge effect on the longitudinal energy spread: variation of the longitudinal energy spread with the beam current.

$q$ parameter for beam current of $1 \mu \mathrm{A}$. As expected, for $\mathrm{rf}$ amplitudes less than $1 \mathrm{kV}(q \leq 0.15)$, the energy spread decreases with rf amplitude. However beyond $1 \mathrm{kV}$, an increase of the energy spread occurs. This increase is not explained by the effect of the rf voltage on the cooling (i.e., on the $\Delta E$ ) rather the derivative effect of the rf field at the RFQ exit is used to explain it. The derivative of the transverse rf field at the RFQ exit gives rise to a longitudinal $\mathrm{rf}$ field which acts on the longitudinal velocities distribution of the cooled ions via its random characteristic. Thereafter, it results in a degradation of this distribution and consequently in growth of the energy spread.

The minimum energy spread obtained with rf amplitude around $1 \mathrm{kV}$ shows the competition between the cooling effect of rf voltage and the degrading effect of longitudinal rf field. At this minimum, $\Delta E$ is around $3.7 \mathrm{eV}$ with a transmission of $38 \%$. For a transmission of at least $60 \%$, the longitudinal energy spread is around $5 \mathrm{eV}$.

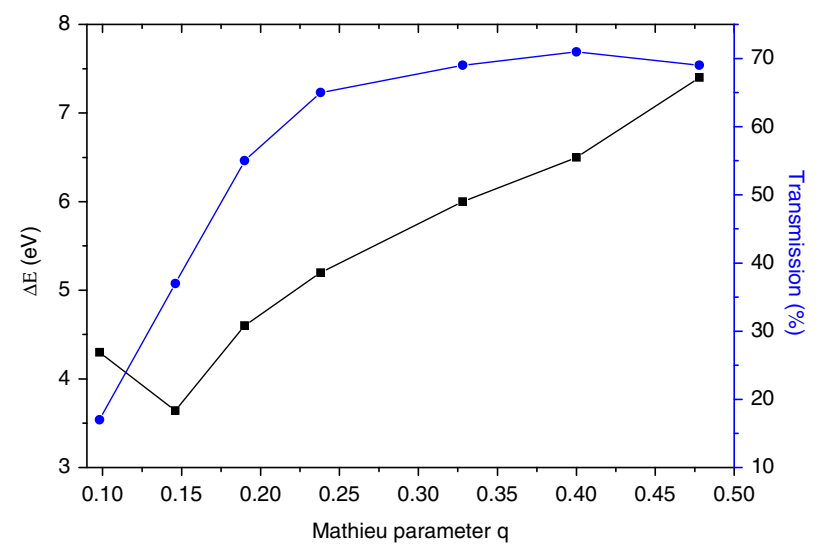

FIG. 13. The rf voltage amplitude effect on the longitudinal energy spread: variation of both the energy spread and its corresponding transmission as a function of the Mathieu parameter $q$.

\section{DISCUSSION}

From the measured data and experience obtained in the above investigations, the following conclusions can be drawn.

The new rf system presented above allows reaching highest rf parameters, up to $9 \mathrm{kV}$ of amplitude and $9 \mathrm{MHz}$ of frequencies, without being limited by any breakdown problem. In such a condition, the space charge effects can be overcome adequately.

The SHIRaC optics system and the appropriate voltages applied to the injection part electrodes show a good efficiency in capturing highest beam currents and transmitting more than $70 \%$ of incoming ions for currents going up to $1 \mu \mathrm{A}$.

For the best cooling condition with SHIRaC, $140 \mathrm{eV}$ of input energy, 2.5 $\mathrm{Pa}$ of RFQ pressure, Mathieu parameter $q=0.4$ (rf amplitude of $2.8 \mathrm{kV}$ and frequency of $4.5 \mathrm{MHz}$ ) and $16 \mathrm{~V} / \mathrm{m}$ of guiding de voltage are sufficient and results in geometrical transverse emittance having low values not exceeding $2.5 \pi \mathrm{mm}$ mrad with beam currents up to $1 \mu \mathrm{A}$.

The only disadvantage of this prototype emerges from the large values of the longitudinal energy spread. This is explained by the derivative effect of the rf field at the RFQ exit for amplitudes more than $1 \mathrm{kV}$.

Both simulations [16] and experiments show close results, in terms of transmission and cooled beam parameters, with intensities never handled so far. For example, at the optimum cooling conditions of $1 \mu \mathrm{A}$ beam, the simulation results are of $67 \%$ of transmission, $2.5 \pi \mathrm{mm} \mathrm{mrad}$ of emittance and $5.9 \mathrm{eV}$ of energy spread and their respective experimental results are of $70 \%$ $(2.3 \pm 0.7 \pi \mathrm{mm} \mathrm{mrad})$ and $(6.5 \pm 0.7 \mathrm{eV})$.

\section{CONCLUSION}

Before building this SHIRaC prototype, the main challenge was to overcome the space charge stemming from the high beam currents. According to numerical simulation presented in Ref. [16], the space charge effects emerge mainly at the RFQ exit region and contribute in degrading both the transmission and the beam quality. But, the large degradation of the energy spread with high beam current, and even with low beam current, could not be explained by these simulations. However, the present experimental studies show that the derivative of the rf confinement field can produce, at the RFQ exit, a longitudinal rf field that can be more degrading than the space charge. The latter is responsible for both transmission reduction and emittance growth but the former acts mainly to enlarge the longitudinal energy spread.

The longitudinal energy spread of the cooled beam is too high considering the expected value which is around $1 \mathrm{eV}$. Therefore, an isobaric purification of this cooled beam by the HRS will become difficult and a development of the extraction part will be necessary. To that end, a miniature 
RFQ should be installed at the RFQ exit [22]. This miniature RFQ aims to avoid the degradation of the cooled beam quality by the degrading effects and to guide these beams to a region where their energy is in few tens $\mathrm{eV}$, so that they can resist any degrading effects.

\section{ACKNOWLEDGMENTS}

We would like to thank Professor G. Ban for continuous encouragement and support. The teams of electronic, vacuum system and mechanical design at L.P.C Caen (France) are gratefully acknowledged for their kind assistance in the development of the project.

[1] M. Lindroos, Review of ISOL-type radioactive beam facilities, in Proceedings of the 9th European Particle Accelerator Conference, Lucerne, 2004 (EPS-AG, Lucerne, 2004).

[2] S. Gales, SPIRAL 2 at GANIL: Next generation of ISOL facility for intense secondary radioactive ion beams, Nucl. Phys. A834, 717c (2010).

[3] Letter of intent for SPIRAL 2.

[4] M. Lewitowicz, The SPIRAL 2 project, Nucl. Phys. A805, 519 (2008)

[5] M. Lewitowicz, Status of the SPIRALl 2 project, Acta Phys. Pol. B 42, p0877 (2011).

[6] E. Minaya Ramirez, S. Cabaret, and D. Lunney, The ion circus: A novel circular Paul trap to resolve isobaric contamination, Nucl. Instrum. Methods Phys. Res., Sect. B 266, 4460 (2008).

[7] The DESIR facility, Letter of intents for SPIRAL 2, 2006.

[8] B. Blanc et al., DESIR: The SPIRAL2 low-energy beam facility, technical proposal for SPIRAL2 instrumentation, 2008, pp. 1-102.

[9] D. Toprek and T. Kurtukian-Nieto, DESIR high resolution separator at GANIL (France), Nucl. Tech. Rad. 27, 346 (2012).

[10] T. Kurtukian-Nieto et al., SPIRAL2/DESIR high resolution mass separator, Nucl. Instrum. Methods Phys. Res., Sect. B 317, 284 (2013).

[11] G. Bollen and S. Schwarz, Penning trap mass measurements on rare isotopes status and new developments, J. Phys. B 36, 941 (2003).

[12] M. Mukherjee et al., ISOLTRAP: An on-line Penning trap for mass spectrometry on short-lived nuclides, Eur. Phys. J. A 35, 1 (2008).

[13] I. Podadera, New developments on preparation of cooled and bunched radioactive ion beams at ISOL-Facilities: The ISCOOL project and the rotating wall cooling, Ph.D. thesis, Universitat Politècnica de Catalunya, Barcelona, Spain, 2006.

[14] J. Dilling et al., Mass measurements on highly charged radioactive ions, a new approach to high precision with TITAN, Int. J. Mass Spectrom. 251, 198 (2006).

[15] R. B. Moore and O. Gianfrancesco, Improving isotope separator performance by beam cooling, Nucl. Instrum. Methods Phys. Res., Sect. B 204, 557 (2003).
[16] R. Boussaid, G. Ban, J. F. Cam, and C. Vandamme, Simulations of high intensity ion beam RFQ Cooler for DESIR/SPIRAL2: SHIRaC, JINST 9, P07009 (2014).

[17] M. D. Lunney and R. B. Moore, Cooling of mass-separated beams using a radio-frequency quadrupole ion guide, Int. J. Mass Spectrom. 190/191, 153 (1999).

[18] A. Nieminen et al., On line Ion Cooling and Bunching for Collinear Laser Spectroscopy, Phys. Rev. Lett. 88, 094801 (2002).

[19] J. Szerypo, D. Habs, S. Heinz, J. Neumayr, P. Thirolf, A. Wilfart, and F. Voit, MAFFTRAP: ion trap system for MAFF, Nucl. Instrum. Methods Phys. Res., Sect. B 204, 512 (2003).

[20] D. A. Dah, SImion 3D V8.0 User Manual (Idaho National Engineering Laboratory, Idaho Falls, 2000).

[21] R. Boussaid, Study and development of buffer gas rf quadrupole cooler for radioactive and very intense ion beams, Ph.D. thesis, Caen Basse-normandie University, France, 2012.

[22] T. Kim, Buffer gas cooling of ions in a radio-frequency quadrupole ion guide: A study of the cooling process and cooled beam properties, Ph.D. thesis, McGill University, 1997.

[23] O. Gianfrancesco, F. Duval, G. Ban, R. B. Moore, and D. Lunney, A radio-frequency quadrupole cooler for highintensity beams, Nucl. Instrum. Methods Phys. Res., Sect. B 266, 4483 (2008).

[24] P. K. Ghosh, Ion Traps (Clarence Press, Oxford, UK, 1995).

[25] C. Bachelet, M. Block, G. Bollen, R. B. Moore, and S. Schwarz, Towards cooling of high intensity ion beams, Hyperfine Interact. 173, 195 (2006).

[26] O. Gianfrancesco, Ion dynamics in a linear high field RFQ trap, Ph.D. thesis, Department of Physics, McGill University, Montreal, Canada, 2005.

[27] M. D. N. Lunney, F. Buchinger, and R. B. Moore, The temperature of buffer gas cooled ions in a Paul trap, J. Mod. Opt. 39, 349 (1992).

[28] W. Paul, Electromagnetic traps for charged and neutral particles, Rev. Mod. Phys. 62, 531 (1990).

[29] F. Herfurth et al., A linear radio-frequency ion trap for accumulation, bunching and emittance improvement of radioactive ion beams, Nucl. Instrum. Methods Phys. Res., Sect. A 469, 254 (2001).

[30] E. Raymond, March, An introduction to quadrupole Ion trap mass spectrometry, J. Mass Spectrom. 32, 351 (1997).

[31] D. J. Douglas et al., Linear ion traps in mass spectrometry, Mass Spectrom. Rev. 24, 1-29 (2005).

[32] S. Heinz, J. Äystö, D. Habs, S. Hegewisch, J. Huikari, A. Nieminen, S. Rinta-Antila, M. Schumann, and J. Szerypo, A radio-frequency ring electrode cooler for low-energy ion beams, Nucl. Instrum. Methods Phys. Res., Sect. A 533, 239 (2004).

[33] Instruction manual, Pepper-pot emittance meter, DREEBIT. V1.0 (2011)

[34] S. Jolly et al., Data acquisition and error analysis for pepper-pot emittance measurements, in Proceedings of the 9th European Workshop on Beam Diagnostics and Instrumentation for Particle Accelerators, Basel, Switzerland, 2009 (PSI, Basel, 2009), WEOA03. 